\title{
Carotid artery \& jugular vein cannulation
}

\section{Surgical narrative}

The goal of this survival surgical procedure is to successfully implant functioning carotid artery and jugular vein catheters in a mouse. All surgeries are conducted in a surgical suite specifically designated for rodent surgery. The surgeon wears clean scrubs, shoes designated only for use in the vivarium, a disposable paper gown, a hair bonnet, a face mask, and sterile gloves. The mouse is obtained from an approved vendor and has met the Institute's 72hour acclimation requirement prior to surgery or was bred on site. For the purpose of this narrative, a 15 week old male mouse is assumed. First, the surgical area is prepared by placing out all instruments, gauze, cotton swabs, suture, and syringes necessary for the procedure on to a sterile drape. This creates the sterile field. These items have been sterilized by autoclave or purchased pre-sterilized. Items that cannot withstand autoclaving such as the catheters and the device used to externalize the catheters are sterilized in a gas sterilizer using ethylene oxide. Each catheter is attached to a syringe containing 100U heparin/ $\mathrm{ml}$ saline. Additionally, two 27-gauge needles are set out and bent 90 degrees at the beveled tip. These will act as introducers to assist proper insertion of the catheters into the vessels.

Before anesthetizing the mouse, he is injected with an analgesic $(0.05 \mathrm{mg} / \mathrm{kg}$ buprenorphine, subcutaneously) for pre-operative pain management as required by the IACUC. The mouse is placed in an induction chamber in which the flow rate of isoflurane anesthesia is set to between 2 and 3percent. The oxygen remains at $1 \mathrm{~L} / \mathrm{min}$ while the percentage of inhaled isoflurane is adjusted accordingly throughout the procedure. To ensure the animal is in the correct surgical plane of anesthesia, constant attention is paid to the animal's respiratory pattern and response to stimuli. The animal should not appear overly sedated with heavy or labored breathing. Alternately, he should not have shallow or rapid breathing. A toe pinch test is performed on the mouse by pinching a hind foot. There should be no movement in response to the toe pinch. Once the mouse is in the correct surgical plane of anesthesia, he is taken to a pre-operative preparation area in the surgical suite and an area between the base of his skull and the interscapular region is promptly shaved. Then the area extending from the lower jaw to the top of the rib cage and between the clavicles is also shaved. The mouse is returned back to the isoflurane anesthesia induction chamber to ensure that he is in the correct surgical plane of anesthesia.

Next, the mouse is placed in the surgical area. In addition to the laid out instruments and supplies, this area consists of a stainless steel plate that sits on top of a warming surface to maintain the mouse's body temperature during the procedure. The surgical area also consists of a stereomicroscope. Sterile ophthalmic lubricant is applied onto both eyes to prevent drying during the surgery. The animal is placed in a supine position and his head is secured into a nose cone that is connected to the isoflurane anesthesia machine. All four limbs are taped down using $3 \mathrm{M}$ surgical tape. To perform aseptic preparation of the surgical incision area, a cotton swab is used to scrub the shaved neck area in a circular motion with Betadine (povidone-iodine) scrub followed by $70 \%$ isopropyl alcohol. This is repeated three times and finished with Betadine solution which is left to dry. Next, the animal is covered with sterile drape, revealing his prepared neck area.

\author{
Volume 4 Issue I - 2016
}

\author{
Emily King BS \\ Sanford-Burnham Medical Research Institute, USA
}

Correspondence: Emily King, BS, Research Associate I , Sanford-Burnham Medical Research Institute at Lake Nona, Cardiometabolic Phenotyping Core, 6400 Sanger Road, Orlando, FL 32827, USA, Tel (407) 745 2000, Extn 6007, Email eking@sanfordburnham.org

Received: July 08, 2016 | Published: November 10, 2016

With a pair of forceps, the shaved area of skin is lifted under the mouse's chin. Scissors are then used to make a $15 \mathrm{~mm}$ midline incision along the neck of the mouse. For catheterization of the carotid artery, the trachea and sternomastoid muscle are exposed and the carotid artery is isolated by gently teasing off any connective tissue with forceps. Care is taken not to disturb the opaque white vagus nerve cord that runs parallel to the carotid artery. Once the carotid artery is isolated, the cephalic end is ligated with 7-0 silk suture. The bifurcation of the carotid artery is used as a reference point at which to ligate the vessel. Next, the caudal end of the artery is loosely knotted and held taught with the weight of a hemostat to disrupt the blood flow. The tip of a bent 27 gauge needle is inserted into the taught carotid artery. Then, the tip of the catheter is slid under the beveled bent needle and the catheter is inserted until its collar reaches the introduction site of the artery. The loose ligature on the caudal end is tightened with a knot to secure the catheter in place. Then, the catheter is secured to the cephalic end using the ends of the cephalic ligature with a knot. Both loose ends of the suture are trimmed. To confirm that the catheter is sampling, pulsatile movement of blood within the tip of the catheter is observed. Blood flow is also observed when withdrawing on the syringe connected to the catheter. Any blood sampled from the mouse is administered back carefully as to not inject substantial heparinized saline into the animal, just enough to flush the catheter line. Lastly, the catheter is clamped with a hemostat. The syringe is removed and replaced with a stainless steel plug. For catheterization of the jugular vein, forceps are used to lift the skin hand and a $10 \mathrm{~mm}$ incision is made with scissors using the right hand approximately $5 \mathrm{~mm}$ to the right of the first arterial incision. The same procedure is repeated with regards to isolating, introducing the catheter into the vessel, and securing the catheter in to place. However, there is no vagus nerve cord or bifurcation present to use as indicators. Additionally, pulsatile movement of blood within the catheter is not usually seen and only occasionally is sampling blood from the vein with the syringe possible. To confirm proper placement of the venous catheter, the catheter line is flushed with $100 \mathrm{u} / \mathrm{ml}$ heparinized saline and no leaks around the vessel should be observed. Once it is clamped with a hemostat, this catheter is also capped with a stainless steel plug when the syringe is removed. Next, the surgical drape is folded and the mouse is turned over in a prone position. The shaved site is prepped in the same 
manner previously described. New sterile gloves are donned and the sterile drape is replaced. With scissors, a $5 \mathrm{~mm}$ incision is made between the shoulder blades.

Using straight forceps, a subcutaneous tunnel is made down the neck region of the mouse on his right side and the venous catheter is pulled through such that it exits from the area between his shoulder blades. This is repeated on the mouse's left side such that the arterial catheter also exits from between the shoulder blades. The lines of the catheters are gently pulled taught so there is no extra slack of the lines within the mouse. Once the catheters are externalized, there sterile drape is folded and the mouse is placed in a supine position. His limbs are secured with $3 \mathrm{M}$ surgical tape. New sterile gloves are donned and the sterile drape is replaced. The ventral incisions are sutured using 6-0 silicon coated braided silk suture with a $3 / 8$ inch $12 \mathrm{~mm} \mathrm{C}-1$ cutting edge needle in a continuous running suture. Precautions are taken to not puncture the catheters with the cutting needle while suturing. The sterile drape is folded and mouse in then placed in a prone position. New sterile gloves are donned and the sterile drape is replaced. Both catheter lines are clamped with a hemostat. The surgical steel plugs are removed and the catheters are connected to a MASA. The MASA is a homemade device consisting of tubing secured in a dollop of hardened medical grade silicone that allows for stability and easy access to the catheters once the mouse is conscious. The MASA is secured in to place by closing the dorsal incision using 6-0 silicon coated braided silk suture with a $3 / 8$ inch $12 \mathrm{~mm} \mathrm{C}-1$ cutting edge needle using a simple interrupted pattern.

Finally, the isoflurane and oxygen are turned off. $0.05 \mathrm{mg} /$ $\mathrm{kg}$ buprenorphine analgesic is administered subcutaneously postoperatively per the IACUC protocol. His body weight is recorded along with any remarkable observations such as blood loss on his surgery cage card. The mouse in placed in lateral recumbency in a warmed cage with the bedding removed. In approximately 2 minutes, once the animal is ambulatory, the mouse is then singly housed on a thermal barrier in the vivarium. The animal is weighed daily and allowed to lose no more than $20 \%$ of his body weight for consideration as a candidate for experimentation. The catheters are checked for patency regularly. Additionally, the mouse is checked daily for signs of pain, distress, and suffering by noting any changes in behavior and appearance. The animal should appear active and curious. Observances are made for lethargy, hunched body posture, a rough unkempt fur coat, and signs of dehydration. A good indicator of a dehydrated mouse is done by performing a tent test in which the skin over his shoulder blades is picked up with the forefinger and thumb. It should return to normal position quickly and not stay in a tented or bunched position. Another indication of dehydration is the appearance of recessed or sunken-in eyes. Occasionally self-mutilation can occur. The incision sites are observed for redness and inflammation or any weeping and oozing which can indicate a sign of infection. If the animal is found to exhibit the indicators mentioned above he is recommended for termination from the study and possibly early euthanasia. The attending Veterinary Technician on site is notified of these observations as well as the responsible party from the laboratory that is conducting the research study. In addition to the surgeon's monitoring, the animal is identified as a surgery animal and monitored by the vivarium staff on weekends and holidays.

\section{Acknowledgements}

None.

\section{Conflict of interest}

Author declares that there is no conflict of interest. 